\title{
Multiple primary malignancies of six organs in a Japanese male patient: A case report
}

\author{
YUTA MUTO, KOICHI SUZUKI, TAKAHARU KATO, KOSUKE ICHIDA, YUJI TAKAYAMA, TARO FUKUI, \\ NAO KAKIZAWA, FUMIAKI WATANABE, YUJI KANEDA, HIROSHI NODA and TOSHIKI RIKIYAMA
}

Department of Surgery, Saitama Medical Center, Jichi Medical University, Omiya-ku, Saitama 330-8503, Japan

Received November 26, 2018; Accepted February 18, 2019

DOI: $10.3892 /$ mco.2019.1819

\begin{abstract}
As a result of recent advances in diagnostic techniques and treatment modalities, the number of patients diagnosed with multiple primary malignancies has been increasing. We report the case of a 79-year-old male with multiple primary malignancies of three histological types in six different organs: Stomach, prostate, colon, urinary bladder, facial skin and pancreas, in chronological order. The first malignancy was upper gastric cancer diagnosed in 1998. The second and third malignancies were prostate cancer and ascending colon cancer, which were diagnosed in 2010. The fourth malignancy was bladder cancer diagnosed in 2011. The fifth and sixth malignancies were squamous cell skin cancer of the right cheek and intraductal papillary mucinous carcinoma (IPMC), respectively, diagnosed in 2014. The gastric cancer, colon cancer, bladder cancer, skin cancer and IPMC were surgically resected. The prostate cancer was treated by anti-androgen therapy. The patient died of local recurrence of IPMC in August 2016. Although multiple primary malignancies are not uncommon, diagnosis of six primary malignancies in a single patient, as reported in the present study, is extremely rare. It is important to understand the characteristics of multiple primary malignancies in order to administer suitable treatment and determine relevant follow-up plans for patients with cancer.
\end{abstract}

Correspondence to: Dr Yuta Muto, Department of Surgery, Saitama Medical Center, Jichi Medical University, 1-847 Amanuma-cho, Omiya-ku, Saitama 330-8503, Japan

E-mail: yutamuto@jichi.ac.jp

Abbreviations: IPMC, intraductal papillary mucinous carcinoma; MPM, multiple primary malignancy; AC, adenocarcinoma; UC, urothelial carcinoma; SCC, squamous cell carcinoma; IPMN, intraductal papillary mucinous neoplasm; CRC, colorectal cancer

Key words: MPM, gastric cancer, prostate cancer, colon cancer, bladder cancer, skin cancer, IPMC

\section{Introduction}

Multiple primary malignancies (MPMs) are defined as more than two primary cancers detected in an individual patient. The classic criteria for the diagnosis of a second primary malignancy were proposed by Warren and Gates in 1932 and include (i) histologic confirmation of malignancy in both the index and secondary tumor; (ii) the two malignancies must be anatomically separated by normal mucosa; and (iii) the possibility of the second primary malignancy being a metastasis from the index tumor must be excluded. MPMs can be classified as either synchronous or metachronous based on their timing of diagnosis (1). According to the Surveillance, Epidemiology, and End Results Program Coding and Staging Manual 2004, synchronous tumors are defined as multiple tumors diagnosed within 2 months of the original/initial diagnosis, and metachronous tumors are multiple tumors or lesions that occur more than 2 months after the original/initial diagnosis (2).

Recent progress in diagnostic techniques and treatment modalities has improved the outcomes of each organ malignancy and resulted in an increase in the number of patients diagnosed with multiple primary malignancies during long-term follow-up (3). Consequently, the risk of developing MPMs is a common problem for cancer survivors. We report a rare case involving a patient with malignancies of three histological types in six different organs, namely adenocarcinoma (AC) of the stomach, prostate, colon, pancreas; a urothelial carcinoma (UC) of the urinary bladder; and a squamous cell carcinoma (SCC) of the facial skin.

\section{Case report}

A 79-year-old male was referred to our hospital for further evaluation of a pancreatic mass on January 2014. He drank a glass of shochu (20 grams of alcohol) a day for 50 years and smoked 20 cigarettes a day for 30 years until he stopped smoking in 2004. His father was diagnosed with lung cancer and his mother was diagnosed with pancreatic cancer.

In March 1998 he was diagnosed with upper gastric cancer and underwent a total gastrectomy and splenectomy with D2 lymph node dissection at a nearby hospital at the age of 63. The pathological diagnosis was moderately differentiated AC, pT3NOM0 (stage IIA), according to the UICC 
Table I. List of malignancies in the present patient.

\begin{tabular}{|c|c|c|c|c|c|c|c|}
\hline \multirow[b]{2}{*}{ Month and year } & \multirow[b]{2}{*}{ Organ } & \multirow[b]{2}{*}{ Histology } & \multicolumn{3}{|c|}{ TNM Staging } & \multirow[b]{2}{*}{ UICC stage } & \multirow[b]{2}{*}{ Treatment } \\
\hline & & & $\mathrm{T}$ & $\mathrm{N}$ & $\mathrm{M}$ & & \\
\hline March 1998 & Stomach & Tubular adenocarcinoma & 3 & 0 & 0 & IIA & $\begin{array}{l}\text { Total gastrectomy } \\
\text { Adjuvant chemotherapy }\end{array}$ \\
\hline March 2010 & Prostate & $\begin{array}{l}\text { Poorly differentiated } \\
\text { adenocarcinoma }\end{array}$ & $1 \mathrm{c}$ & 0 & 0 & I & MAB \\
\hline November 2010 & Colon & Tubular adenocarcinoma & 3 & 0 & 0 & IIA & $\begin{array}{l}\text { Right hemicolectomy } \\
\text { Adjuvant chemotherapy }\end{array}$ \\
\hline July 2011 & Bladder & $\mathrm{UC}$ & Tis & 0 & 0 & 0 & TURBT \\
\hline January 2014 & Skin & $\mathrm{SCC}$ & 1 & 0 & 0 & I & Resection \\
\hline January 2014 & Pancreas & IPMC & 1 & 0 & 0 & IA & Pancreaticoduodenectomy \\
\hline
\end{tabular}

Table II. Previous studies of multiple primary malignancies occurring in five or six different organs.

\begin{tabular}{|c|c|c|c|c|c|c|c|}
\hline Author, year & Country & Age & Sex & Organs, listed chronologically & Family history & Prognosis & Refs. \\
\hline Jones, 1976 & UK & 37 & M & Duo, Sma, Kid, Col, Pan & Unknown & Unknown & (4) \\
\hline Uchida et al, 1987 & Japan & 62 & $\mathrm{~F}$ & Eso, Lym, Ute, Sto, Ora, Pha & No & Unknown & - \\
\hline Sommers et al, 1988 & USA & 57 & $\mathrm{~F}$ & Skin, Col, Kid, Ova, Ute & Unknown & Unknown & (5) \\
\hline Hashimoto et al, 1997 & Japan & 59 & M & Rec, Bla, Sto, Col, Liv, Lung & Unknown & Unknown & - \\
\hline Martin-Granizo et al, 1997 & Spain & 61 & $\mathrm{~F}$ & Lar, Bla, Pro, Ora, Ton & Unknown & Alive & (6) \\
\hline Yakushiji et al, 1999 & Japan & 71 & M & Rec, Bla, Sto, Col, Liv, Lung & Yes & Alive & (7) \\
\hline Moriyama et al, 2008 & Japan & 76 & $\mathrm{~F}$ & Sto, Kid, Bla, Rec, Col, Sma & Yes & Alive & - \\
\hline Watanabe et al, 2008 & Japan & 80 & $\mathrm{~F}$ & Col, Ute, Duo, Sto, Mam, Sma & Yes & Unknown & - \\
\hline Cercato et al, 2008 & Italy & 75 & $\mathrm{~F}$ & Ute, Thy, Rec, Col, Bro & Unknown & Alive & (8) \\
\hline Komiyama et al, 2012 & Japan & 46 & $\mathrm{~F}$ & Ova, Ute, Col, Rec, Lung & Yes & Alive & (9) \\
\hline Mukaiyama et al, 2014 & Japan & 58 & M & Glo, Kid, Bla, Ora, Pro, Eso & No & Dead & (10) \\
\hline Murakami et al, 2014 & Japan & 73 & M & Lar, Eso, Sto, Pro, Pha, Lung & Unknown & Alive & - \\
\hline Hashimoto et al, 2014 & Japan & 60 & M & Eso, Sto, Glo, Sma, Col, Lung & No & Alive & - \\
\hline Present case, 2018 & Japan & 79 & M & Sto, Pro, Col, Bla, Skin, Pan & No & Dead & \\
\hline
\end{tabular}

-, Japanese article from the Japan Medical Abstracts Society Database; Duo, duodenum; Sma, small intestine; Kid, kidney; Col, colon; Pan, pancreas; Eso, esophagus; Lym, malignant lymphoma; Ute, uterus; Sto, stomach; Ora, oral cavity; Pha, pharynx; Ova, ovary; Rec, rectum; Bla, bladder; Liv, liver; Pro, prostate; Ton, tongue; Mam, mammary gland; Thy, thyroid; Bro, bronchus; Glo, glottis; M, male; F, female.

TNM classification, 7th edition. Adjuvant chemotherapy was performed with mitomycin $\mathrm{C}$ and oral uracil/tegafur for 18 months. In March 2010 he underwent prostate cancer screening and was diagnosed with adenocarcinoma of the prostate with Gleason score $5+5$, according to the Gleason grading system. He was treated with maximum androgen blockade therapy. In November 2010, stenotic ascending colon cancer was detected by colonoscopy performed at the previous hospital. He underwent right hemicolectomy with D3 lymph node dissection. The pathological finding was well differentiated AC, pT3N0M0, stage IIA, according to the UICC TNM classification, 7th edition. As the tumor was large $(40 \mathrm{~mm})$ and stenotic, he was administered adjuvant chemotherapy with oral uracil/tegafur and oral leucovorin for 1 year. In July 2011, cystoscopy revealed a bladder tumor and the patient underwent transurethral resection of bladder tumor in August 2011. The pathological finding was UC of the urinary bladder, high-grade, pTisNOM0 (stage 0), according to the UICC TNM classification, 7th edition. In January 2014 he noticed a mass on his right cheek. Dermatologists resected that mass completely. The pathological finding was a squamous cell skin cancer of right cheek, pT1N0M0 (stage I), according to the UICC TNM classification, 7th edition. An abdominal CT scan in January 2014 for follow-up after colon cancer treatment revealed dilatation of the tortuous pancreatic duct and a $10-\mathrm{mm}$ tumor at the pancreatic head. Although he 


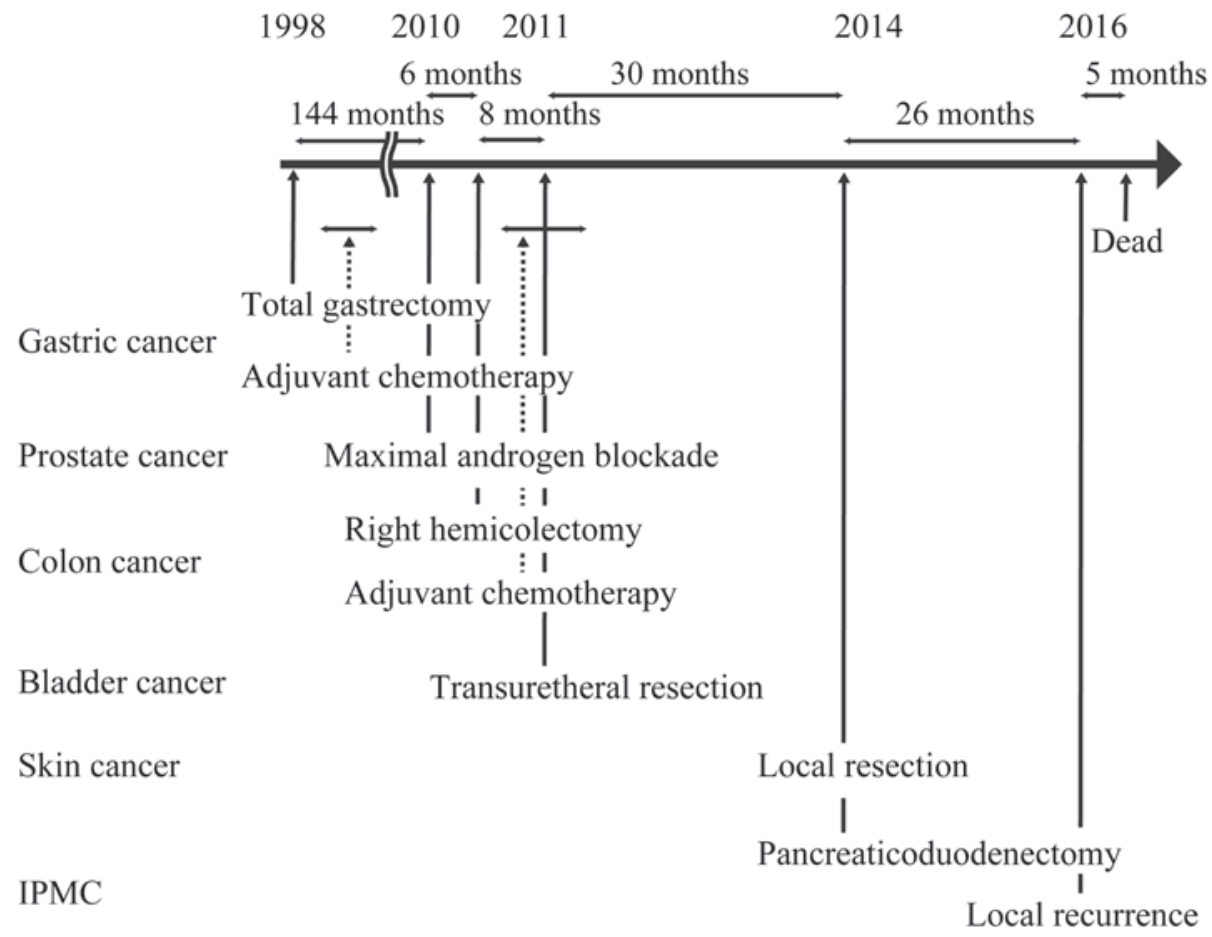

Figure 1. Time course of the patient with multiple primary malignancies and treatments for each cancer. IPMC, intraductal papillary mucinous carcinoma.

had no symptoms, the tumor was suspected to be intraductal papillary mucinous neoplasm (IPMN). He was referred to our hospital for further investigation and treatment. Endoscopic retrograde cholangiopancreatography and cytology were performed and he was diagnosed with intraductal papillary mucinous carcinoma (IPMC). We performed pancreaticoduodenectomy with D1 lymph node dissection. The pathological finding was IPMC of the main pancreatic duct in pancreas head, well differentiated, pT1, N0, M0 (stage IA), according to the UICC TNM classification , 7th edition. After the surgery follow-up was conducted by an abdominal CT scan performed every 6 months and local recurrence of IPMC appeared in March 2016. After receiving best supportive care, he died of cancer in August 2016.

The six primary malignancies are listed in chronological order in Table I, and the schema of this patient time course is described on Fig. 1.

\section{Discussion}

The case presented here is exceedingly rare in view of the number of primary malignancies and their combination. We found only 13 papers that reported multiple malignancies of five or six different organs from a search of PubMed and the Japan Medical Abstracts Society databases (Table II) (4-10). Besides, we could not find any reports of this particular combination of MPMs to date. Nine cases were reported from Japan and 4 cases were from other countries. There is no difference between Japanese and worldwide MPMs cases, e.g. age, sex, organs.

Demandante et al (11) reviewed 1,104,269 cancer patients in the literature and reported that the prevalence of multiple primary malignant neoplasms ranges between 0.734 and $11.7 \%$. They stated that multiple primary malignant neoplasms might occur more frequently than expected by random chance. They also noted that the incidence of developing MPMs increased with increasing age (11). The precise risk of MPMs in Japan is unknown. Tabuchi et al reported that metachronous second primary cancers developed in $3.8 \%$ of cancer patients after a median follow-up of 2.5 years and the 10 -year cumulative risk was estimated as $13.0 \%$ for those who first developed cancer at 60-69 years of age (12).

It is difficult to estimate the risk of sextuple cancers. However, several investigators have reported patients with MPMs for each type of cancer. We previously reported that MPMs at extracolonic sites were identified in 117 of 1,111 patients $(10.5 \%)$ with colorectal cancer (CRC) and that gastric cancer had the highest incidence $(44.4 \%, 52$ of 117) among all MPMs. The rarest combination was that of CRC and skin cancer, which was found in only 1 of 117 patients with MPMs (3). For patients with gastric cancer, Takeuchi et al (13) reported that MPMs were observed in 109 patients (25.1\%): 40 (9.2\%) with synchronous and $76(18.2 \%)$ with metachronous MPMs. The most common malignancy was colorectal cancer (22.8\%). These data seem to suggest that CRC and gastric cancer share common risk factors. In a previous study, microsatellite instability (14), translocation of TP53 $(15,16)$, and ageing $(17,18)$ were identified as shared risk factors between CRC and gastric cancer. Cercato et al reported that most MPMs arise in the respiratory, gastrointestinal, and genitourinary systems and one of the most common malignancies is prostate cancer (33-64\% of all MPMs in a male patient series) (8). Regarding IPMN, it has been generally accepted that patients with IPMN have an increased risk for MPMs (19,20). We previously reported the relationship between IPMN and MPMs, showing that MPMs were more frequent in patients with malignant (50.0\%) IPMNs than in those with benign disease $(21.6 \%)$. We suggested that IPMNs, especially malignant IPMNs, have an increased risk for the development of neoplastic disease (21). 
Powell et al (22) studied the prognosis of patients with synchronous MPMs and metachronous MPMs. The median survival time was 49 months in the synchronous group compared with 115 months in the metachronous group (adjusted hazard ratio $0.50, \mathrm{p}<0.001$ ). This is somewhat expected, as incidental malignancies found on imaging studies such as those used for staging are often in an earlier stage (22). In the present case, the patient unfortunately had a recurrence of IPMC despite metachronous malignancies.

Several factors affect the etiology of MPMs, including lifestyle (e.g., tobacco, alcohol, diet), environment (e.g., contaminants, occupation), and host factors (e.g., genetics, immune function, hormonal interactions) (23). Tobacco smoking is one of the major causes of multiple primary malignancies, with strong well-established associations with tumors of the lung, oral cavity, pharynx, nasal cavity, larynx, esophagus, stomach, pancreas, colorectum, liver, kidney, ureter, urinary bladder, uterine cervix, and ovary, and myeloid leukemia (24). Patients who continue to smoke after development of a first smoking-related primary cancer have a substantially increased risk of developing smoking-related secondary primary cancers (25). Alcohol intake has been implicated as one of the causes of upper aerodigestive tract cancers such as tumors of liver, breast, and colorectum (26). Moreover, synergistic effects of tobacco and alcohol exist for upper aerodigestive tract cancers $(23,27)$. In the present case, smoking might be associated with the incidence of colon cancer, bladder cancer and IPMN. Similarly, alcohol consumption may have contributed to increase the risk of acquiring colon cancer.

We also should take into account genetic factors that contribute to diseases, such as Lynch syndrome. The incidence of MPMs has been reported to be high in patients with Lynch syndrome. However, previous studies have suggested that the frequency of Lynch syndrome in Japan is low, ranging from 0.15 to $0.2 \%$ compared with up to $5 \%$ in Western countries (28). Among the cases of MPMs listed in Table II, four had family histories of cancer and three did not; family history was unknown for the remaining cases. In the present case, the patient did not meet the Amsterdam II Criteria or the Revised Bethesda Guidelines. Other major hereditary gastrointestinal cancer syndromes are familial adenomatous polyposis (FAP), Li-Fraumeni syndrome, Hereditary diffuse gastric cancer (29). FAP is an autosomal dominant disease that is classically characterized by the development of hundreds to thousands of adenomas in the rectum and colon during the second decade of life (30). The patient did not have the typical FAP phenotype of polyposis. The diagnosis of FAP was unlikely. Li-Fraumeni syndrome is a cancer predisposition syndrome caused by a germline mutation of the TP53 gene (31), and hereditary diffuse gastric cancer is characterized by an accumulation of diffuse type gastric cancer in the family pedigree (32). These syndromes were unlikely in the present case, since the patients was elder male and the combination of MPMs was not typical of these hereditary cancer syndromes. Although we could not exclude completely the possibility of hereditary cancer syndromes as we did not perform germline exome sequencing, the MPMs of the present patient might have been sporadic in nature, rather than hereditary.

In conclusion, patients with cancers typically undergo far more testing than the general population, accounting for the high detection rate of additional malignancies. As a result of increased detection of early cancers and development of new therapeutic strategies, the prognosis for cancer patients has steadily improved. This improvement in prognosis has led to an increased incidence of MPMs. In the near future, molecular biologic techniques might help to diagnose MPMs earlier. It is important to understand the characteristics of MPMs in order to administer suitable treatment and determine relevant follow-up plans for cancer patients.

\section{Acknowledgements}

Not applicable.

\section{Funding}

No funding was received.

\section{Availability of data and materials}

The datasets used and analyzed during the current study are available from the corresponding author on reasonable request.

\section{Authors' contributions}

YM and KS conceived the study. YM was involved in the acquisition of clinical documents and drafting the manuscript. KS and TK supervised the writing of the manuscript. FW, YK, HN and TR performed the surgery. TK, KI, YT, TF, NK, FW, YK, HN and TR treated the patient. All authors read and approved the final version of the manuscript.

\section{Ethics approval and consent to participate}

Written informed consent for surgery, chemotherapy and publication was obtained from the patient.

\section{Patient consent for publication}

Written informed consent was obtained from the patient for publication of this case report.

\section{Competing interests}

The authors declare that they have no competing interests.

\section{References}

1. Warren S and Gates O: Multiple primary malignant tumors: A survey of the literature and a statistic study. Am J Cancer 16: 1358-1414, 1932.

2. Johnson CH (ed): SEER Program Coding and Staging Manual 2004, Revision 1. National Cancer Institute, NIH Publication, Bethesda, MD, 2004.

3. Kato T, Suzuki K, Muto Y, Sasaki J, Tsujinaka S, Kawamura YJ, Noda H, Horie H, Konishi F and Rikiyama T: Multiple primary malignancies involving primary sporadic colorectal cancer in Japan: Incidence of gastric cancer with colorectal cancer patients may be higher than previously recognized. World J Surg Oncol 13: 23, 2015.

4. Jones P: Letter: Five separate malignancies in one patient. BMJ 1: 1533, 1976.

5. Sommers GM,Logan S and Camel HM: Six independent neoplasms in one woman. A case report. J Reprod Med 33: 82-83, 1988. 
6. Martin-Granizo R, Naval L, Castro P, Goizueta C and Muñoz M: Quintuple cancers: Report of a case with triple cancers in the head and neck. J Craniomaxillofac Surg 25: 153-157, 1997.

7. Yakushiji H, Mukai S, Matsukura S, Sato S, Ogawa A, Sasatomi E and Miyazaki K: DNA mismatch repair deficiency in curatively resected sextuple primary cancers in different organs: A molecular case report. Cancer Lett 142: 17-22, 1999.

8. Cercato MC, Colella E, Ferraresi V,Diodoro MG and Tonachella R: Report of two cases of quintuple primary malignancies and review of the literature. Anticancer Res 28: 2953-2958, 2008.

9. Komiyama S, Nishio E, Ichikawa R, Miyamura H, Kawamura K, Komiyama M, Nishio Y and Udagawa Y: Asymptomatic synchronous quintuple primary cancers. Gynecol Obstet Invest 74: 324-328, 2012.

10. Mukaiyama Y, Suzuki M, Morikawa T, Mori Y, Takeshima Y, Fujimura T, Fukuhara H, Nakagawa T, Nishimatsu H, Kume $\mathrm{H}$ et al: Multiple primary malignant neoplasms of the glottis, renal pelvis, urinary bladder, oral floor, prostate, and esophagus in a Japanese male patient: A case report. World J Surg Oncol 12: 294, 2014.

11. Demandante CG, Troyer DA and Miles TP: Multiple primary malignant neoplasms: Case report and a comprehensive review of the literature. Am J Clin Oncol 26: 79-83, 2003.

12. Tabuchi T, Ito Y, Ioka A, Miyashiro I and Tsukuma H: Incidence of metachronous second primary cancers in Osaka, Japan: Update of analyses using population-based cancer registry data. Cancer Sci 103: 1111-1120, 2012.

13. Takeuchi D, Koide N, Komatsu D, Okumura M, Suzuki A and Miyagawa S: Relationships of obesity and diabetes mellitus to other primary cancers in surgically treated gastric cancer patients. Int J Surg 12: 587-593, 2014.

14. Yun HR, Yi LJ, Cho YK, Park JH, Cho YB, Yun SH, Kim HC Chun HK and Lee WY: Double primary malignancy in colorectal cancer patients--MSI is the useful marker for predicting double primary tumors. Int J Colorectal Dis 24: 369-375, 2009.

15. Uchino S, Noguchi M, Ochiai A, Saito T, Kobayashi M and Hirohashi S: p53 mutation in gastric cancer: A genetic model for carcinogenesis is common to gastric and colorectal cancer. Int J Cancer 54: 759-764, 1993.

16. Sawai T, Nanashima A, Tsuji T, Yamaguchi $H$, Yasutake $T$, Nakagoe T, Ayabe $\mathrm{H}$ and Tagawa Y: Instability of chromosome 17 and the p53 locus in non-familial colorectal cancer with multiple primary malignancies. J Exp Clin Cancer Res 20: 401-405, 2001.

17. Schatzkin A, Baranovsky A and Kessler LG: Diet and cancer. Evidence from associations of multiple primary cancers in the SEER program. Cancer 62: 1451-1457, 1988.

18. Saito S, Hosoya Y, Togashi K, Kurashina K, Haruta H, Hyodo M, Koinuma K, Horie H, Yasuda Y and Nagai H: Prevalence of synchronous colorectal neoplasms detected by colonoscopy in patients with gastric cancer. Surg Today 38: 20-25, 2008.

19. Sugiyama M and Atomi Y: Extrapancreatic neoplasms occur with unusual frequency in patients with intraductal papillary mucinous tumors of the pancreas. Am J Gastroenterol 94: 470-473, 1999.
20. Larghi A, Panic N, Capurso G, Leoncini E, Arzani D, Salvia R, Del Chiaro M, Frulloni L, Arcidiacono PG, Zerbi A, et al: Prevalence and risk factors of extrapancreatic malignancies in a large cohort of patients with intraductal papillary mucinous neoplasm (IPMN) of the pancreas. Ann Oncol 24: 1907-1911,2013.

21. Kato T, Alonso S, Noda H, Miyakura Y, Tsujinaka S, Saito M, Muto Y, Fukui T, Ichida K, Takayama Y, et al: Malignant, but not benign, intraductal papillary mucinous neoplasm preferentially associates with prior extrapancreatic malignancies. Oncol Rep 35: 3236-3240, 2016.

22. Powell S, Tarchand G, Rector T and Klein M: Synchronous and metachronous malignancies: Analysis of the Minneapolis Veterans Affairs (VA) tumor registry. Cancer Causes Control 24: 1565-1573, 2013.

23. Travis LB, Rabkin CS, Brown LM, Allan JM, Alter BP, Ambrosone CB, Begg CB, Caporaso N, Chanock S, DeMichele A, et al: Cancer survivorship--genetic susceptibility and second primary cancers: Research strategies and recommendations. J Natl Cancer Inst 98: 15-25, 2006

24. Begg CB, Zhang ZF, Sun M, Herr HW and Schantz SP: Methodology for evaluating the incidence of second primary cancers with application to smoking-related cancers from the Surveillance, Epidemiology, and End Results (SEER) program. Am J Epidemiol 142: 653-665, 1995.

25. Do KA, Johnson MM, Lee JJ, Wu XF, Dong Q, Hong WK, Khuri FR and Spitz MR: Longitudinal study of smoking patterns in relation to the development of smoking-related secondary primary tumors in patients with upper aerodigestive tract malignancies. Cancer 101: 2837-2842, 2004.

26. Pöschl G and Seitz HK: Alcohol and cancer. Alcohol Alcohol 39: $155-165,2004$.

27. Schottenfeld D: Alcohol as a co-factor in the etiology of cancer. Cancer 43 (Suppl 5): 1962-1966, 1979.

28. Yamamoto S, Yoshimura K, Ri S, Fujita S, Akasu T and Moriya Y: The risk of multiple primary malignancies with colorectal carcinoma. Dis Colon Rectum 49 (Suppl 10): S30-S36, 2006.

29. Hata K, Yamamoto Y, Kiyomatsu T, Tanaka T, Kazama S, Nozawa H, Kawai K, Tanaka J, Nishikawa T, Otani K, et al: Hereditary gastrointestinal cancer. Surg Today 46: 1115-1122, 2016.

30. Half E, Bercovich D and Rozen P: Familial adenomatous polyposis. Orphanet J Rare Dis 4: 22, 2009.

31. Correa H: Li-Fraumeni Syndrome. J Pediatr Genet 5: 84-88, 2016.

32. Fitzgerald RC, Hardwick R, Huntsman D, Carneiro F, Guilford P, Blair V, Chung DC, Norton J, Ragunath K, Van Krieken JH, et al; International Gastric Cancer Linkage Consortium: Hereditary diffuse gastric cancer: Updated consensus guidelines for clinical management and directions for future research. J Med Genet 47: 436-444, 2010 . 